\title{
THREE-DIMENSIONAL ULTRASOUND IMAGING OF THE EYE
}

\author{
DÓNAL B. DOWNEY ${ }^{1,3}$, DAVID A. NICOLLE ${ }^{2}$, MORRIS F. LEVIN ${ }^{1}$ \\ and AARON FENSTER ${ }^{1,3}$ \\ London, Ontario
}

\begin{abstract}
SUMMARY
We assessed whether an inexpensive, three-dimensional (3D) ultrasound (US) imaging system could produce clinically useful 3D images, without causing patient discomfort. Five patients were examined. The 3D US system consisted of a transducer holder containing a mechanical motor, and a microcomputer. During data acquisition the transducer was mechanically rotated for 22 seconds, while 200 two-dimensional (2D) US images were collected and formed into a 3D image by the computer. The 3D image was viewed on the computer monitor. The 3D images correlated with the clinical and radiological findings. The new perspectives were helpful in diagnosing eye abnormalities and no patient discomfort occurred. The device was easy to use. It is concluded that, as good-quality $3 D$ and $2 D$ US images were produced quickly, with no patient discomfort, and the device is inexpensive, uncomplicated, and easily attached to existing ultrasound machines, it will probably be useful in clinical practice.
\end{abstract}

Conventional ophthalmic ultrasonography is an established diagnostic technique that is essential for the clinical practice of ophthalmology. ${ }^{1}$ Both brightness mode (B-mode) imaging, which gives information about the topographic nature of ocular and orbital lesions, and amplitude mode (A-mode) imaging, which gives information about the size and nature of lesions, are widely used. ${ }^{2}$ Increasingly, colour Doppler imaging is being utilised to assess blood flow in the blood vessels of the eye and orbit. $^{3-5}$ Although it is generally agreed that ultra-

From: ${ }^{1}$ Department of Diagnostic Radiology and Nuclear Medicine, and ${ }^{2}$ Department of Ophthalmology, University Hospital, University of Western Ontario, London, Ontario N6A 5A5, Canada; ${ }^{3}$ The Tom Lawson Family Imaging Research Laboratories, The John P. Robarts Research Institute, 100 Perth Drive, London, Ontario N6A 5K8, Canada.

Correspondence to: Dr Dónal Downey, Department of Diagnostic Radiology and Nuclear Medicine, University Hospital, 339 Windermere Road, London, Ontario N6A 5A5, Canada. sound imaging is cost-effective, ${ }^{6}$ we believe technical improvements are needed before its full diagnostic potential is realised.

Though orbital anatomy and pathology are threedimensional (3D), the B-mode ultrasound data are presented to the examiner in a two-dimensional (2D) format. This is regardless of whether the information is viewed directly from the ultrasound monitor, from videotape, or from photographic film or paper. To interpret the data, the diagnostician mentally integrates multiple 2D images into a 3D impression of the anatomy and pathology being examined. ${ }^{7}$ While experienced examiners are often extremely skilful at developing this mental 3D representation of the anatomy, the process itself is inefficient and requires considerable learning on behalf of the operator. No matter how assiduously one performs the ultrasound examination, or how well trained one is, it is probable that at least some of the available diagnostic information is lost during this process. For example, when the scan is performed by a technician and only a few 2D ultrasound photographic images are later reviewed by the clinician, inaccuracies may occur because out-of-plane features have not been recorded. In addition, it is often difficult to localise the exact plane in which any 2D ophthalmic image was obtained. This makes locating the exact position of an anomaly difficult. To reproduce a particular image plane at a later time, or to follow up some orbital pathologies with 2D US is complex, especially if the abnormalities have tapered or indistinct margins. ${ }^{8}$

Standard 2D images are usually obtained in an antero-posterior direction, either in a transverse, axial or longitudinal plane, or in a plane parallel or slightly oblique to, one of these planes. ${ }^{7}$ Some ocular pathologies may be more appropriately viewed from a mediolateral orientation or a more oblique orientation, and these perspectives may not be adequately achieved using conventional $2 \mathrm{D}$ US. 
Many authors have concluded that a 3D display of the ultrasound information would correct some of these weaknesses and improve diagnostic ability. Many attempts have been made to develop 3D systems suitable for use in different parts of the body. ${ }^{9-12}$ Some endeavours have been reported in the ophthalmology literature, ${ }^{13-17}$ though none, as yet, have been widely implemented. These systems were generally unsuccessful either because the 3D imaging devices were too slow due to immature technology, or because they required complex instrumentation. Previously, 3D ultrasound images were acquired slowly and the structures that were clinically reviewed were generated manually by tracing the contour of the organ on each 2D image. These images were stacked on top of each other to create a 'wire frame' representation. These wire frames were then assembled by computer into a 3D image and shaded to produce a surface-rendered representation. ${ }^{13,15}$ This technique is labour-intensive and excludes important ultrasound information about the interior of the organ. The images appear artificial, making interpretation difficult.

Recently it has become possible to purchase fast, powerful and inexpensive microcomputers, and we believe that now is an appropriate time to reevaluate methods of $3 \mathrm{D}$ ultrasound information display. The following were considered important criteria in the design of this new system. All diagnostically relevant ultrasound information from the original $2 \mathrm{D}$ images should be preserved in the reconstructed 3D image. It should also be possible to view and manipulate the $3 \mathrm{D}$ volume interactively in distinct 2D planes, orientated in any direction. The 2D 'slices' obtained from the 3D image should be similar to those obtained in a standard 2D examination. 'Slicing' of the 3D image should occur rapidly, data acquisition and reconstruction times should be short, and the system should be relatively inexpensive.

On the basis of this approach we constructed a prototype 3D US imaging system. The purposes of this preliminary study were twofold: (1) to evaluate whether this new system could produce accurate images in vivo, and (2) to ensure that the technique would be acceptable to patients.

\section{MATERIALS AND METHODS}

Five patients with different ocular pathologies - a choroidal melanoma, a post-traumatic vitreous haemorrhage and retinal detachment, a simple retinal detachment, a treated retinal detachment and a retroorbital tumour - were evaluated with the 3D ultrasound imaging system. All gave informed consent and the study received approval from the university human ethics committee. The prototype 3D US system consisted of a standard, commercially available, end-firing, sector type mechanical transducer (Ophthascan Mini B $10 \mathrm{MHz}$ transducer, Biophysic Medical, Clermont-Ferrand, France), coupled with a Macintosh Quadra 840A microcomputer (Apple, Cupertino, CA). To obtain a 3D image, the ultrasound transducer was inserted into a specially designed motorised transducer holder (Fig. 1). The holder was held by the examiner in a manner that allowed the transducer tip to be closely coupled with the closed eyelid of the supine patient. ${ }^{18}$ Copious amounts of ultrasound coupling gel facilitated good visualisation of structures in the orbit (Figs. 3, 4). The 2D B-mode ultrasound image was optimised by altering the time-gain curve, field-of-view and overall gain settings. When a focal abnormality in the eye was being viewed (e.g. melanoma) the lesion was positioned in the centre of the field of view of the $2 \mathrm{D}$ image. This ensured that the ultrasound beam was perpendicular to the object being examined, allowing optimum measurements to be made.

The patient kept eye and body still, and the examiner held the transducer holder as steadily as possible (Figs. 3, 4). The motor was activated for 22 seconds, causing the transducer to rotate about its own axis through $200^{\circ}$ (Fig. 2, top). After each $1^{\circ}$ angular movement, the resulting ultrasound B-mode video image was captured by a frame grabber (Precision Digital Images, Redmond, WA) and stored in the microcomputer (Fig. 2, bottom left). Care was taken to ensure that the cable from the ultrasound transducer never became twisted, and that it remained clear of the patient's face at all times.

Immediately after the scan, the 200 B-mode images were reviewed on the microcomputer monitor sequentially, and evaluated to ensure that each had been accurately recorded and that no patient or examiner motion had occurred during the examination. If any motion was evident, another data acquisition was obtained and the original one was discarded. Two satisfactory 3D data sets were obtained on each patient.

The acquired images were reconstructed into a 3D image (Fig. 2, bottom right) using a computer program developed in our laboratory. Data reconstruction time was 140 seconds.

The viewing software, also developed in our laboratory, allows the data to be viewed in different ways. The simplest involves presenting the acquired 200 B-mode 2D US images to the examiner on the computer screen. This is the easiest method for most beginners to interpret, as the data are exactly the same as they would see in a standard examination, except that there are many more 2D images presented for review. The 3D display software allows the examiner to rotate the 3D image in any direction, and 'slice' it interactively to obtain the desired 


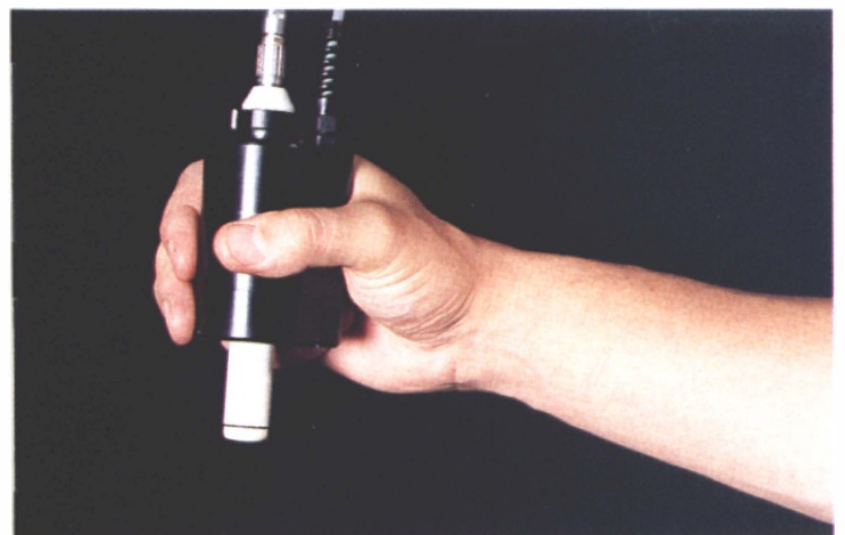

Fig. 1. Motorised transducer holder. The white ultrasound transducer has been coupled with the black motorised holder.

perspective. Viewing is also possible with other commercially available $3 \mathrm{D}$ viewing software. The microcomputer monitor (Figs. 5, 6) has two display windows: the $3 \mathrm{D}$ volume image window and a labelled cartoon of the eye. The 3D volume image is a cube containing all the diagnostic ultrasound information from the eye. With a computer mouse, this image can be easily rotated in any direction and then 'sliced' away to reveal the desired anatomy (Figs. 5, 6). After 'slicing' a certain distance into the $3 \mathrm{D}$ image, one can activate another face of the 3D image and 'slice' into that face in exactly the same manner. In addition, a facility is available to 'slice' obliquely in any plane (Fig. 5c).

The 'slice' displayed on the monitor changes almost instantaneously with movements of the

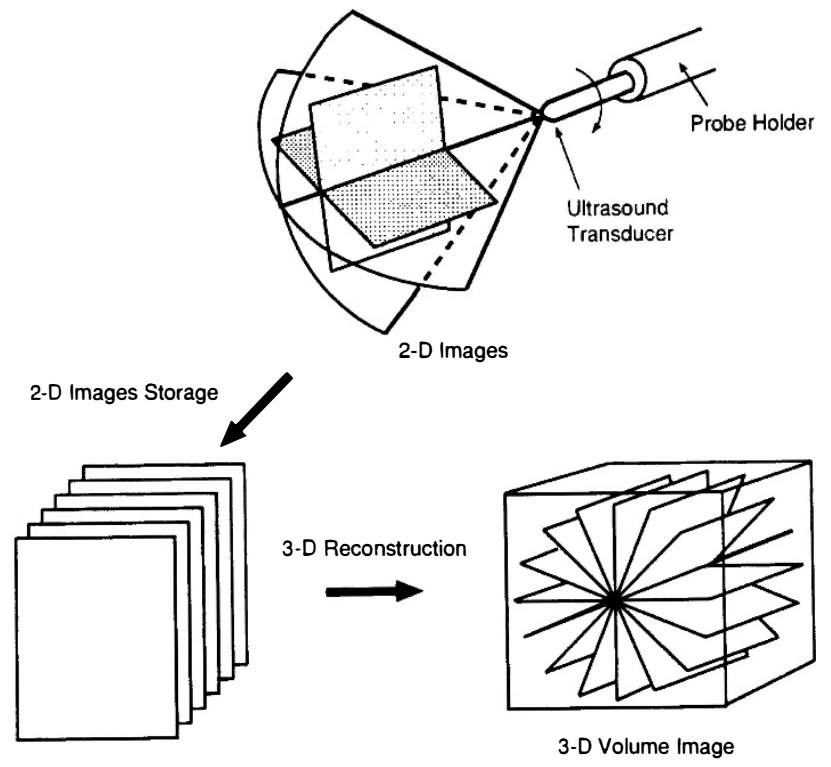

Fig. 2. Diagrammatic representation of $3 D$ image formation. The transducer rotates about its own axis (top); 3 of the 200 different $2 D$ B-mode images obtained during a standard scan are shown. The images are stored in order (bottom left) and then reassembled in a cylinder (bottom right) ready for viewing at the workstation.

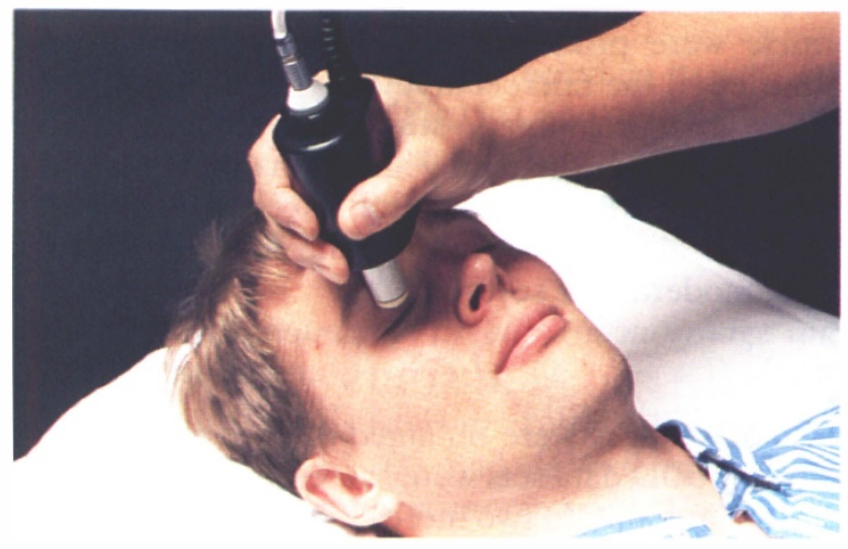

Fig. 3. $3 D$ patient scanning. The coupled holder and transducer are applied to the closed eyelid using ultrasound coupling gel.

mouse, so the impression the evaluator gets is very similar to that obtained while scanning a patient in real time. The orientation of the cartoon corresponds with the orientation of the 3D image, and the 'slice' of the 3D image being shown is also graphically marked on the cartoon (Figs. 5, 6). This ensures that one can always tell the location and orientation of any given 'slice'. The response time of the cartoon graphic is virtually instantaneous.

Measurement of area is achieved by having the user outline a structure in a given 'slice' by dragging the mouse (Fig. 5c), and once the desired structure has been outlined the computer determines its area. By moving to adjacent 'slices' known distances away (e.g. $1 \mathrm{~mm}$ ) and repeating the outlining process, the volume of the structure is obtained by summing the area in each 'slice' and multiplying that by the distance between 'slices'. The 3D images, as well as selected 'slices' and views from the volume, can be archived onto an optical disk which is interfaced to the microcomputer. Other image output devices may be attached to the microcomputer for other forms of archive: for example, an image printer, video tape recorder or slide maker. The images shown in this

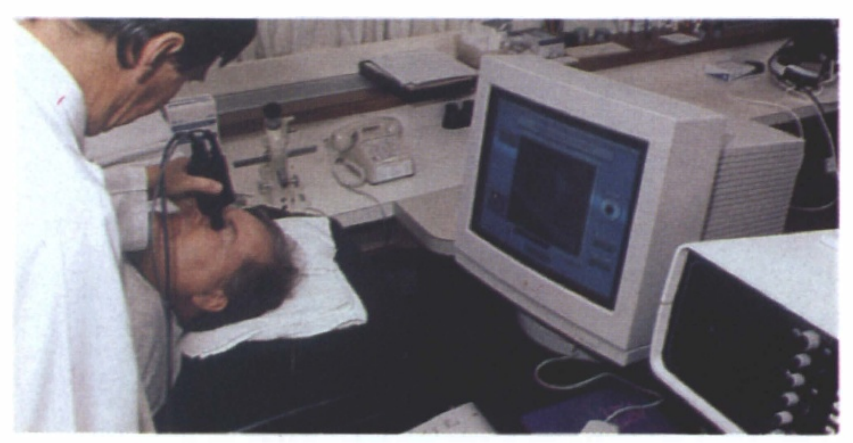

Fig. 4. $3 D$ examination acquisition. The operator is holding the coupled holder/transducer in position on the eyelid while the transducer rotates about its own axis. The $B$-mode ultrasound images are being simultaneously displayed on both the ultrasound monitor and the computer screen. 
article were printed directly from a Sony printer attached to the microcomputer.

For this preliminary study, the images of five patients were evaluated by an ophthalmologist and a radiologist. All patients were asked to grade the level of discomfort during the examination as mild, moderate or severe.

\section{RESULTS}

Two 3D images were obtained in each case. In two cases, two 3D data sets were successfully obtained on the first attempt. In two more cases, a single extra 3D image was required, and in the fifth case two

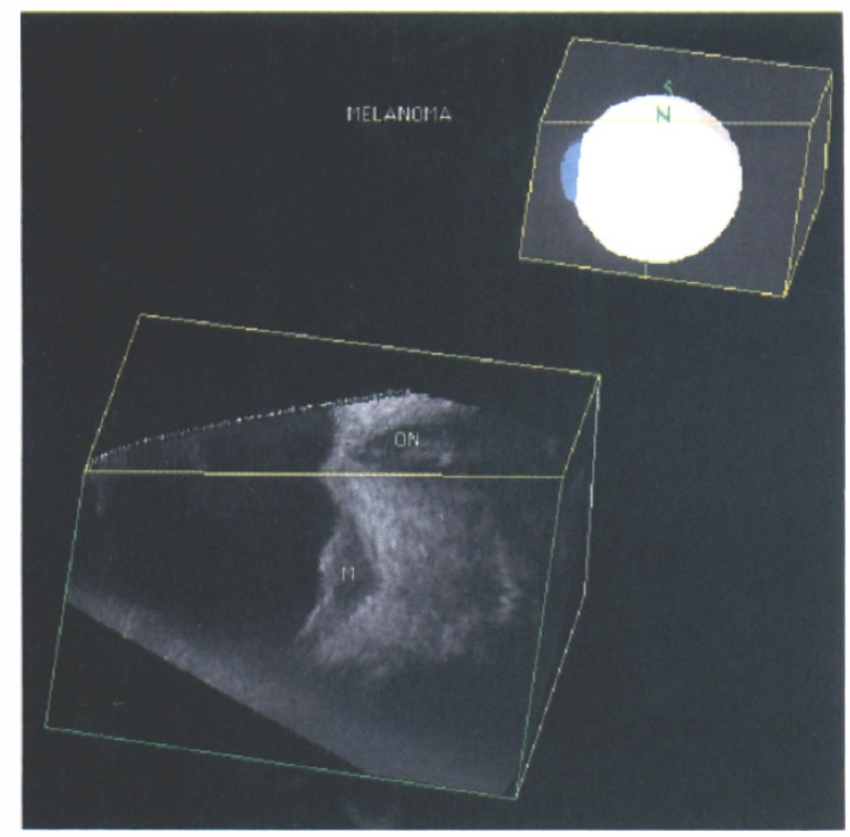

(a)

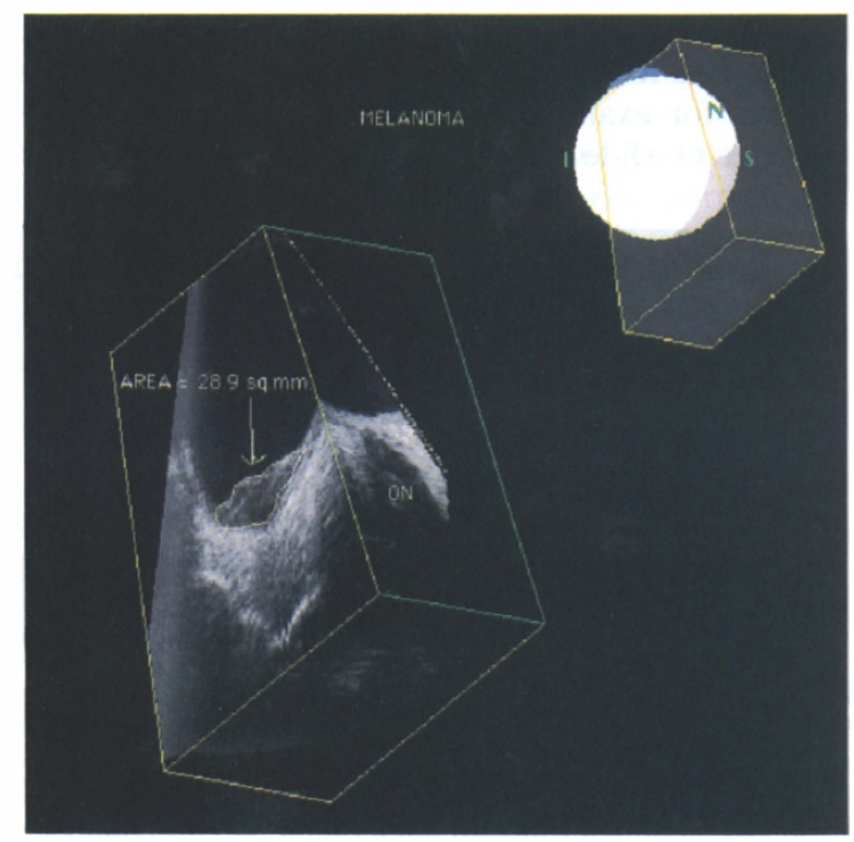

(c) additional 3D image acquisitions were required. The reasons for the extra images were patient motion (three examinations) and examiner motion (one examination) during the patient scanning phase of the examination. All examinations, including patient set-up, scanning review, reconstruction and 3D image examination, were completed within 7 minutes. No discomfort was reported upon direct questioning of each patient.

Regardless of the viewing perspective chosen, the $3 \mathrm{D}$ image conformed as expected with the pathology in each case. The transverse and longitudinal perspectives of the 3D image were the same as had

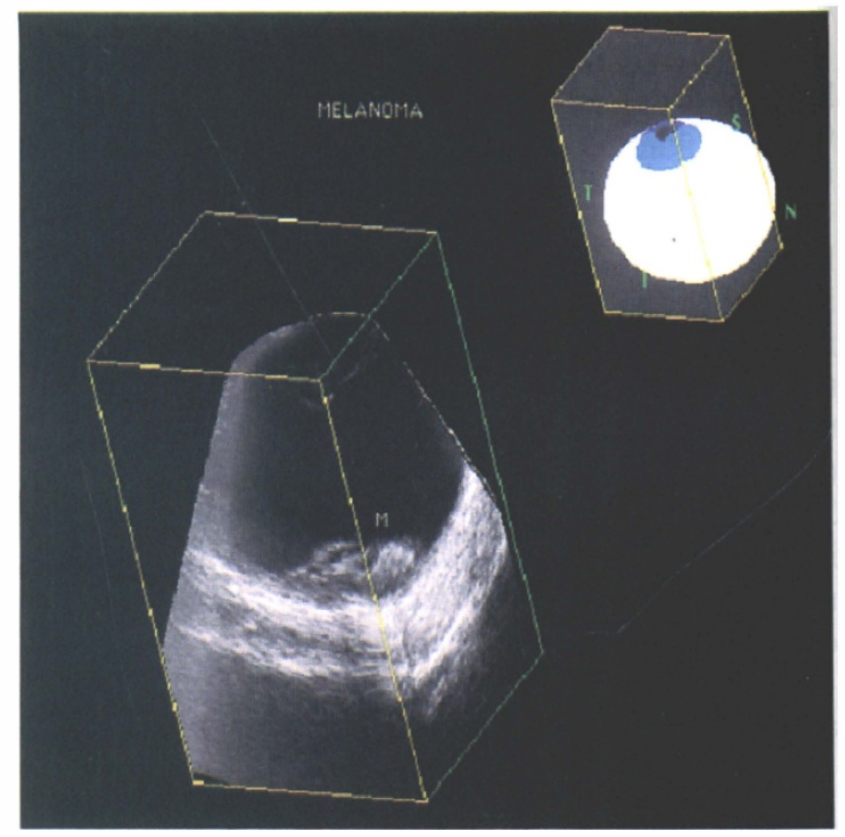

(b)

Fig. 5. Choroidal melanoma. (a) Side view. A melanoma $(M)$ is shown in one plane and the optic nerve $(O N)$ is shown superiorly in a plane at $90^{\circ}$ to the original plane. The cartoon in the upper right corner shows the orientation of the globe. The different surfaces of the cartoon are labelled: $S$, superior; $N$, nasal; $I$, inferior. (b) Different planes and orientation. The same melanoma $(M)$ is shown in a different orientation with the iris lying superiorly. Two faces of the tumour are shown in different planes. The cartoon shows orientation of both the globe and the planes being displayed: $S$, superior; $N$, nasal; $T$, temporal; $I$, inferior. (c) $3 D$ measurement. In a given plane the outline has been mapped out by the operator and the computer has calculated the outlined area. (ON, optic nerve). To measure the tumour volume accurately in $3 D$, the operator moves to a plane parallel to the current plane but $1 \mathrm{~mm}$ away and repeats the measurement and the computer automatically computes the volume. This process continues until the entire tumour is measured. S, superior; $N$, nasal; I, inferior. 


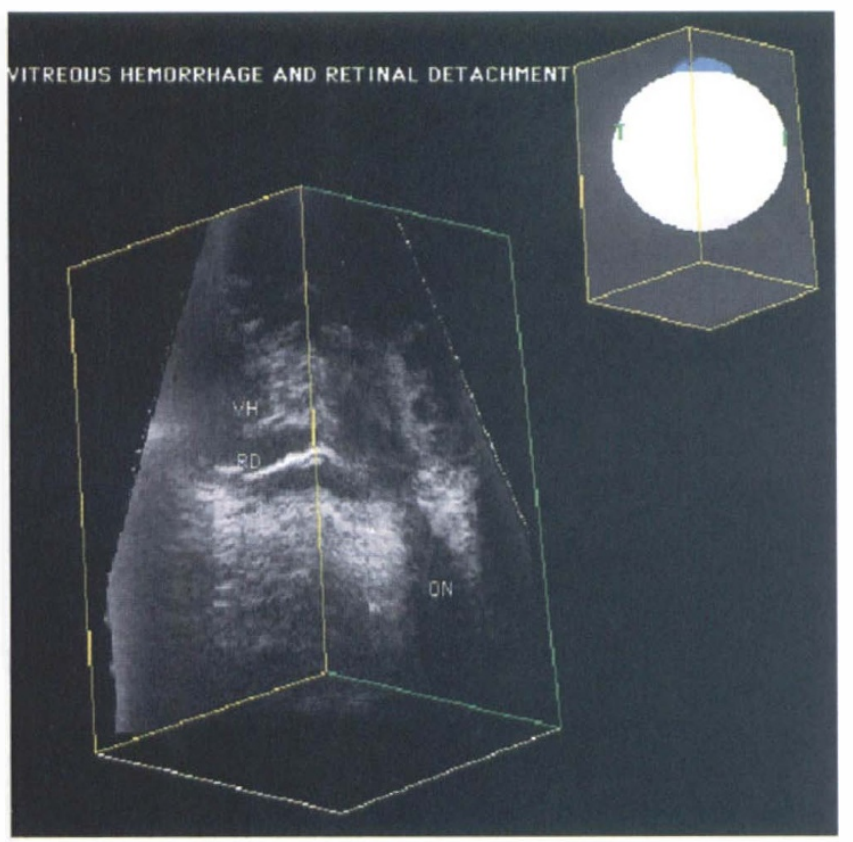

Fig. 6. Vitreous haemorrhage and retinal detachment. In this $3 D$ image, the iris is located superiorly and the optic nerve $(\mathrm{N})$ is inferior. A large vitreous haemorrhage (VH) and an underlying retinal detachment $(R D)$ are shown in two planes. The cartoon shows the orientation and location of the image plane: T, temporal; I, inferior.

been seen on the standard ultrasound. The newer viewing planes also appeared accurate with no obvious artefacts.

In each 3D examination, the optic nerve was clearly shown (Figs. 5 and 6 ) in all cases, and its relationship to the different pathologies was clearly demonstrated (Fig. 5c). The contour of the globe was well preserved. No artefacts thought likely to distort the accurate clinical interpretation of the image were appreciated.

In all five cases, the 3D ultrasound images correlated with the standard 2D ultrasound examinations, both clinically and surgically. There were no positive conventional ultrasound findings that were not appreciated on 3D ultrasound. In one patient with a post-traumatic vitreous haemorrhage (Fig. 6). the location and extent of a retinal detachment were reported to be shown better on 3D than on standard 2D ultrasound by both the ophthalmologist and radiologist. Both reported it easier to obtain the maximum tumour length measurement on $3 \mathrm{D}$. One examiner preferred to view the eye with the sclera on the left of the screen (Fig. 5a) while the other preferred the sclera at the top of the screen (Fig. 5b). Both perspectives were available, and both evaluators were pleased with the variety of perspectives available to them.

Two illustrative cases are described below.

\section{Case 1. Choroidal Melanoma}

A 66-year-old man has been followed for 1 year with a choroidal melanoma in his right eye. 3D ultrasound shows the lesion well (Fig. 5). including its relationship to the optic nerve (Fig. 5a). In addition, the 3D image allowed us to measure the volume of the tumour (Fig. 5c). Note how the tumour can be viewed in different orientations (Fig. 5a-c).

\section{Case 2. Post-traumatic Vitreous Haemorrhage and Retinal Detachment}

A 44-year-old man sustained multiple injuries in a motor-cycle accident. He had an extensive scleral laceration and vitreous haemorrhage that prevented direct visualisation of the retina. 3D ultrasound images showed the vitreous haemorrhage: also the posterior retinal detachment (Fig. 6). This was confirmed by both standard 2D ultrasound and surgery. As the viewing plane in the $3 \mathrm{D}$ image could be tilted in any direction, the evaluators believed this perspective to be superior to the $2 \mathrm{D}$ examination.

\section{DISCUSSION}

It has been two decades since ophthalmic ultrasound was at the forefront of ultrasound technology when the first array scanner was built for ophthalmic use. ${ }^{19}$ Since then, its premier position has been gradually eroded with the newer ultrasound advances being directed to other body parts. Now that 3D ultrasound is being evaluated in viewing other areas, ${ }^{9-12}$ we believe it is important to assess its use in the eye. The eye is well known as an excellent acoustic medium. with no air or calcification in it. It is relatively immobile, with a high fluid content and significant differences in the acoustic impedances of its various components. ${ }^{7.9}$ The eye also has advantages that make it ideal for the use of 3D imaging technology: it is reasonably small, which means the $3 \mathrm{D}$ US images require only moderate amounts of random access memory (RAM), and since there is no cardiac or respiratory motion, the scan acquisition requires no special gating and is completed in 22 seconds.

Clearly, there is a need for a better method of displaying ultrasound information. At present, the best US diagnostic results are achieved by diagnosticians scanning the eye in different planes using 2D technology and building up a 3D image in their minds. ${ }^{7}$ Usually, the longer one scans and the more imaging planes one utilises, the better one understands the pathology. Unfortunately, there is a limit to the length of time one can spend scanning, as some patients become restless. With this device, the total patient examination time is short: all our examinations took less than 7 minutes and data acquisition time was 22 seconds. We would expect to halve the total examination time with increased experience. The patient with post-traumatic changes reported significantly more discomfort during the conven- 
tional $2 \mathrm{D}$ scan than during the $3 \mathrm{D}$ scan. If this feature is confirmed in larger studies, it is likely to speed up patient examination and throughput times in busy clinics.

With this new system, the time available for review is increased indefinitely, and the clinician can spend as much time as needed reviewing the images. In this study, both examiners spent more time than they would normally have done with a conventional 2D examination, evaluating details of the eye on the 3D scan. They believed that having the patient out of the room facilitated them in being more thorough in their assessments. Image evaluation can be done at any time, and cases may be discussed with colleagues long after the patient has left the facility. Fishman et $a l .{ }^{20}$ considered 3D images to be an excellent focus for clinicians to discuss patient diseases. Indeed, as the images are digital and can be transferred by the usual methods of digital data transfer (for example, diskettes, telephone lines and computer networks), remote consultations and discussions are possible.

The display software is easy to learn, with all activity being controlled by a computer mouse. The fact that it allows multiple $2 \mathrm{D}$ 'slices' to be viewed individually means that 'beginners' can relate to the images almost immediately. This is contrary to some previous 3D US systems whose surface-rendered images were very unfamiliar even to ophthalmologists.

Coleman et al. ${ }^{13,17}$ have argued that $3 \mathrm{D}$ rendering of ophthalmic pathology is especially valuable in the globe because it clearly shows the relationship of orbital structures to each other, thus greatly helping the ophthalmologist to plan surgery. In the case of the orbital melanoma, the tumour location was clearly shown relative to other structures in the eye (Fig. 5). The display module allows the information to be displayed in novel planes, some of which may prove more useful than the conventional planes currently used.

3D imaging allows tumours and intraocular haemorrhages to be accurately measured, which is essential for evaluating the prognosis and making appropriate decisions in managing some intraocular tumours. ${ }^{21}$ In evaluating these patients over time there is a need for ultrasound measurements to become more standardised, accurate and repeatable, particularly for the evaluation of lesions with tapering or ill-defined edges. ${ }^{8}$ With conventional 2D ultrasound, reproducing the exact planes on which the original measurements were done may be very difficult. If the entire lesion is preserved in a 3D image, a more precise measurement is likely. A disadvantage of the system is an inability to visualise the entire globe with a single acquisition, so that abnormalities especially in the anterior aspects of the eye may be incompletely visualised or missed completely. To perform a thorough examination of all parts of the globe, we believe that at least three and possibly more data acquisitions in different orientations need to be obtained.

Dynamic information, which is often very helpful in diagnosing such conditions as retinal detachments and vitreous floaters, is not available with the 3D scan. In addition, contact problems between the ultrasound transducer and eyelid are much more likely than with standard ultrasound examinations. During standard examinations subtle changes are made by the examiner to ensure optimal transducer contact. Such minor movements are not possible with the $3 \mathrm{D}$ system as they would interfere with the geometry of the final image.

In conclusion, this inexpensive and uncomplicated prototype $3 \mathrm{D}$ ultrasound imaging device, which can be coupled to any standard ultrasound machine, produced high-quality images quickly and without causing significant patient discomfort. It allows immediate and convenient access to hundreds of $2 \mathrm{D}$ US image planes, in addition to providing $3 \mathrm{D}$ perspectives.

Authors wish to thank S. Tong, MSc, for designing and drawing Fig. 2, and Ms L. Carey for typing the manuscript. Supported by grants from The University Hospital Pooled Research Trust Fund, University Hospital, London, Ontario, Canada, and The Medical Research Council of Canada, Ottawa, Ontario, Canada, grant no. MA-12486.

Key words: Ultrasound, experimental; Ultrasound, ophthalmic.

\section{REFERENCES}

1. Byrne SF, Green RL. Ultrasound of the eye and orbit. St Louis: Mosby Year Book, 1992:ix.

2. Byrne SF, Green RL. Ultrasound of the eye and orbit. St Louis: Mosby Year Book, 1992:15.

3. Erickson SJ, Hendrix LE, Massaro BM, Harris GJ, Lewandowski MF, Foley WD, Lawson TL. Colour Doppler flow imaging of the normal and abnormal orbit. Radiology 1989;173:511-6.

4. Munk P, Downey D, Nicolle D, Vellet AD, Rankin R, Lin DT. The role of colour flow Doppler ultrasonography in the investigation of disease in the eye and orbit. Can J Ophthalmol 1993; 28:171-6.

5. Aburn NS, Sergott RC. Orbital colour Doppler imaging. Eye 1993;7:639-47.

6. Straub WH. Relative strength and limitations of diagnostic imaging studies. In: Manual of diagnostic imaging, 2nd ed. Boston: Little, Brown, 1989: chap.3.

7. Byrne SF, Green RL. Ultrasound of the eye and orbit. St Louis: Mosby Year Book, 1992:17-21.

8. Byrne SF, Green RL. Ultrasound of the eye and orbit. St Louis: Mosby Year Book, 1992:150.

9. Rankin RN, Fenster A, Downey DB, Munk PL, Levin MF, Vellet AD. Three-dimensional sonographic reconstruction: techniques and diagnostic applications. AJR 1993;161:695-702.

10. Richard WD, Grimell CK, Bedigian K, Frank KJ. A method for three-dimensional prostate imaging using transrectal ultrasound. Computerised Medical Imaging and Graphics 1993;17:73-9.

11. Sehgal CM, Broderick GA, Whittington R, Gorniak 
RJT, Arger PH. Three-dimensional US and volumetric assessment of the prostate. Radiology 1994;192:274-8.

12. Kelly IM, Gardener JE, Brett AD, Richards R, Lees WR. Three-dimensional US of the fetus: work in progress. Radiology 1994;162:253-9.

13. Coleman DJ, Woods S, Rondeau MJ, Silverman RH Ophthalmic ultrasonography. Radiol Clin North Am 1992;30:1105-14.

14. Baum G. Orbital lesion localisation by three dimensional ultrasonography. NY State J Med 1961;61:414957.

15. Jensen PK, Hansen MK. Ultrasonographic, threedimensional scanning for determination of intraocular tumour volume. Acta Ophthalmol (Copenh) 1991;69:176-86.

16. Yamamoto Y, Kubota M, Sugata Y, Matsui S, Ito M. Three dimensional ultrasonography of ocular region.
In: Thijssen JM (editor). Ultrasonography in ophthalmology. Dordrecht: Kluwer, 1988:11-8.

17. Coleman DJ, Silverman RH, Rondeau MJ, Lizzi FL. New perspectives: 3D volume rendering of ocular tumours. Acta Ophthalmol Suppl 1992;204:22.

18. Munk PL, Vellet AD, Levin M, Lin DTC, Collyer RT Sonography of the eye. AJR 1991;157:1079-86.

19. Buschmann W. Sophisticated instrumentation and ophthalmic ultrasonography. Acta Ophthalmol Suppl 1992:204:18-21.

20. Fishman EK, Magid D, Ney DR. Three-dimensional imaging. Radiology 1991:181:321-37.

21. Flock M, Gerende JH, Zimmerman LE. The size and shape of malignant melanomas of the choroid and ciliary body in relation to prognosis and histologic characteristics. Trans Am Acad Ophthalmol Otolaryngol 1955:59:740. 